\title{
LE POETE CRITIQUE : DE BAUDELAIRE A VALERY
}

\section{O POETA CRÍTICO: DE BAUDELAIRE A VALÉRY}

\author{
Daniela Lindenmeyer Kunze \\ Universidade Federal de Pernambuco, UFPE, Recife, PE, Brasil \\ Anselmo Peres Alós \\ Universidade Federal de Santa Maria, UFSM, Santa Maria, RS, Brasil
}

Résumé: Dans les études littéraires françaises, on constate la forte présence de textes théoriques et d'essais sur la poésie écrits par des poètes. On remarque une tradition française de poètes critiques qui a comme principal précurseur Charles Baudelaire. Depuis celui-ci, cette écriture critique accompagne la création poétique des principaux poètes français et gagne encore plus de force chez les contemporains. Ces réflexions critiques peuvent apparaître dans l'œuvre d'un poète sous différentes formes : certains poètes ont écrit des textes théoriques, d'autres ont exposé leurs conceptions de la poésie et de la création poétique dans leur correspondance et d'autres finalement les ont transformées en matière première de leur création : ils ont greffé cette préoccupation critique au sein de leur poétique créant une poésie qui parle d'elle-même - la métapoésie.

Mots-clés: Poésie française; poètes critiques; métapoésie.

Resumo: Nos estudos literários franceses, pode-se constatar a forte presença de textos teóricos e de ensaios sobre poesia escritos por poetas. Destaca-se uma tradição francesa de poetas críticos que possui, como principal precursor, Charles Baudelaire. Desde entâo, tal escritura crítica acompanha a criação poética dos principais poetas franceses e ganha ainda mais força junto aos contemporâneos. Tais reflexōes críticas podem aparecer dentro da obra de um poeta sob diferentes formas: certos poetas escreveram textos teóricos, outros expuseram suas concepçóes de poesia e de criação poética em sua correspondência, e outros, finalmente, transformaram essa questão em matéria-prima para suas criaçôes: eles destacam tal preocupação no seio mesmo de suas poéticas, criando uma poesia que fala dela mesma - a metapoesia.

Palavras-chave: Poesia francesa; poetas críticos; metapoesia.

Pour Hugo Friedrich, la rencontre de la création littéraire et de la réflexion critique est « un des phénomènes essentiels des Temps Modernes " (FRIEDRICH, 1999, p. 67) et cette tradition inaugurée par Charles Baudelaire a inspiré directement les poètes Arthur Rimbaud et Stéphane Mallarmé ${ }^{1}$ et, plus tard, Paul Valéry et Pierre Reverdy à porter un regard

${ }^{1} C f$. Igitur, Divagations, Un coup de dés. $C f$. aussi Correspondance complète suivi de 
critique sur la poésie et les autres arts. Rimbaud écrit deux lettres en 1871 où il livre le programme poétique d'une « littérature nouvelle " (LEUWERS, 1998, p. 90). Mallarmé écrit également, entre 1872 et 1898, des essais ainsi qu'une correspondance riche en réflexions critiques sur la poésie et réunit autour de lui une nouvelle génération de poètes très attentifs à ses propos sur la littérature. Parmi ces jeunes poètes, on trouve Paul Valéry qui deviendra l'écrivain critique le plus important du $\mathrm{XX}^{\mathrm{e}}$ siècle. Valéry défend, dans un essai emblématique, la notion de poète critique (VALÉRY, 2002, p. 657691) affirmant que tout poète est aussi un critique et que toute création poétique doit être accompagnée d'un travail de réflexion sur l'écriture. Cette notion ${ }^{2}$ sera renforcée quelques décennies après par le poète contemporain Jean-Michel Maulpoix avec sa théorie d'un lyrisme critique (MAULPOIX, 2004).

Dans la lignée de poètes critiques français, Charles Baudelaire est l'auteur le plus significatif, car il en est le précurseur. Il écrit des essais sur la littérature, la peinture, la musique et fait la critique des ouvrages de ses contemporains. Ces essais sont basés sur l'observation et l'interprétation des œuvres littéraires, des arts plastiques et de la musique ainsi que des commentaires et des analyses sur des événements d'arts. Selon Hugo Friedrich, les réflexions critiques et les commentaires de Baudelaire s'élargissent tellement qu'ils deviennent finalement " des analyses de la conscience de l'époque " (FRIEDRICH, 1999, p. 44) - une sorte de poétique de la modernité. Son œuvre critique devient donc aussi importante que sa poésie, ces textes théoriques sont d'une très grande importance dans la littérature mondiale compte tenu l'influence qu'ils exerceront sur la théorie littéraire et sur quelques-uns des poètes les plus significatifs du XIX et $\mathrm{XX}^{\mathrm{e}}$ siècle :

Un aspect fondamental de Baudelaire est sans doute sa rigueur intellectuelle,
la clarté de sa conscience artistique. Il unit le génie poétique à l'intelligence
critique. Ses réflexions sur le mécanisme de la création poétique se situent
à un niveau aussi élevé que sa poésie elle-même, la dépassant même parfois
[...] Les perspectives qu'il ouvre ainsi ont exercé sur les années à venir une
influence peut-être plus profonde que Les Fleurs du Mal proprement dites
(FRIEDRICH, 1999, p. 44).

Pour Hugo Friedrich, ces réflexions sur la création poétique inaugurent, chez les poètes modernes, le goût de la critique et le besoin

\section{Lettres sur la poésie.}

2 L'appellation de "poète critique ", avec les connotations données par ces deux auteurs, reviendra tout au long de cen travail. 
d'une constante réflexion sur la création poétique. Rimbaud, Mallarmé et Valéry ont tous les trois produits des textes critiques très importants et qui restent encore aujourd'hui des références pour les théoriciens et les poètes :

[...] l'œuvre de Baudelaire donna rapidement naissance en France à des courants différents de ceux du romantisme, beaucoup plus novateurs, qui imprégnèrent les œuvres de Rimbaud, de Verlaine, de Mallarmé. [...] Vers la fin de sa vie, Valéry voyait encore une ligne qui conduisait directement de Baudelaire à lui-même (FRIEDRICH, 1999, p. 43).

Arthur Rimbaud étale ses réflexions critiques sur la poésie, la création poétique et le langage dans certains passages du livre Une saison en enfer et, de manière plus théorique, dans deux lettres écrites en 1871. La première date du 13 mai et est adressée à Georges Izambard, jeune professeur de rhétorique dans le collège de Rimbaud. La seconde est écrite le 15 mai et est adressée à Paul Demeny, un poète de la région. Les deux ne sont publiées qu'en 1912 et 1928 et sont alors appelées Les lettres du voyant. A côté des réflexions critiques sur la poésie et le langage, Rimbaud nous livre sa conception de la poésie moderne et présente son programme poétique pour les poèmes à venir. Ces Lettres deviennent ainsi des textes importants pour comprendre la révolution dans l'écriture opérée par le jeune poète. Daniel Leuwers les considère comme des " textes véritablement fondateurs de la poésie et de la poétique modernes" (LEUWERS, 1998, p. 7). Elles sont également la confirmation "que, pour Rimbaud, la création poétique est inséparable d'une réflexion tout aussi importante sur la poésie " (LEUWERS, 1998, p. 83). Suivant la tradition inaugurée par Baudelaire qui, selon le poète, " est le premier voyant, roi des poètes" (RIMBAUD, 1999, p. 93), Rimbaud fait de la critique et défend ce nouveau rôle du poète qui observe et qui s'efforce de comprendre son propre processus créatif :

[...] j'assiste à l'éclosion de ma pensée : je la regarde, je l'écoute : je lance un coup d'archet : la symphonie fait son remuement dans les profondeurs, ou vient d'un bond sur la scène. [...] La première étude de l'homme qui veut être poète est sa propre connaissance, entière ; il cherche son âme, il l'inspecte, il la tente, l'apprend (RIMBAUD, 1999, p. 88).

Il fait aussi la critique de la poésie romantique dont le grand défaut est, pour lui, l'absence d'un regard critique et objectif sur sa création, d'une 
" véritable prise de conscience du geste créateur " (LEUWERS, 1998, p. 22): "les romantiques, qui prouvent si bien que la chanson est si peu souvent l'œuvre, c'est-à-dire la pensée chantée et comprise du chanteur ?" (RIMBAUD, 1999, p. 87).

Stéphane Mallarmé fait également partie de ces poètes critiques fortement marqués par l'héritage baudelairien. Une des caractéristiques de son œuvre est, selon Friedrich, c'est l'" affirmation de l'égale valeur de la poésie et de la réflexion sur la poésie " (FRIEDRICH, 1999, p. 133). Ces réflexions critiques sont exprimées "sous forme théorique " dans les essais réunis en 1897 dans Divagations (MALLARMÉ, 2003) et dans ses Lettres sur la poésie (MALLARMÉ, 1995) écrites entre 1872 et 1898. Comme son maître Baudelaire, il écrit sur la poésie, sur son expérience en tant que poète, sur la peinture, sur le théâtre et sur d'autres poètes de son époque, parmi lesquels on trouve Verlaine, Rimbaud et Baudelaire.

Dans la préface de l'édition qui réunit sa correspondance, Yves Bonnefoy souligne les spécificités des réflexions critiques de Mallarmé dans l'ensemble de ses lettres. Encore une fois le caractère singulier de cette critique d'écrivain est confirmé par la part de liberté et de création qu'elle contient ${ }^{3}$.

Amoureux de la forme et de la technique, sa poésie arrive à une perfection formelle grâce à " une méditation approfondie sur le langage et l'objet de la poésie » (BRUNEL, 1977, p. 548). De ce fait, chez Mallarmé, la production poétique et la critique sont intimement liées. Elles coexistent et se complètent. Dans la plupart des essais de Divagations, ainsi que dans d'autres textes réunis dans la même édition, Mallarmé emploi un langage proche du poétique, rempli de métaphores et autres figures de langage. On se trouve en face des textes où se confondent parfois critique, rêve et suggestion :

Mon Baudelaire à peine ouvert, je suis attiré dans un paysage surprenant qui vit au regard avec l'intensité de ceux que crée le profond opium. Là-haut, et à l'horizon, un ciel livide d'ennui, avec les déchirures bleues qu'a faites la Prière proscrite. Sur la route, seule végétation, souffrent de rares arbres dont l'écorce douloureusement est un enchevêtrement de nerfs dénudés : leur croissance visible est accompagnée sans fin, malgré l'étrange immobilité de l'air, d'une plainte déchirante comme celle des violons, qui, parvenue à l'extrémité des branches, frissonne en feuilles musicales (MALLARMÉ, 2003, p. 353-354).

${ }^{3}$ Cf. BONNEFOY, Yves. Poèmes (2001) ; L'alliance de la poésie et de la musique (2007); Entretiens sur la poésie (1991); et finalement L'inachevable (2012). 
Dans ces quelques lignes de l'essai consacré à Théophile Gautier, à Théodore de Banville et à Baudelaire, il utilise des images et suggère des sensations pour parler des Fleurs du mal de Baudelaire. Sa critique est, finalement, proche de sa poésie - les deux plutôt basées sur la suggestion que sur la description.

Paul Valéry est l'un des jeunes poètes qui fréquentent la maison de Mallarmé à Paris à partir de 1891, un de "disciples charmés par ses propos sur la poésie et la musique " (LAGARDE; MICHARD, 1967, p. 529). Alors influencé par Mallarmé, mais aussi par Baudelaire, il produit une œuvre critique très significative qui est, selon Mireille Calle-Gruber, plus «forte " que sa poésie : "L'intelligence hors pair fait de lui un grand poéticien : La Méthode de Léonard de Vinci, 1895 [...], Monsieur Teste, 1896, les Cahiers, sont sans doute plus forts que l'œuvre poétique " (CALLEGRUBER, 2001, p. 165). Ses essais sur la poésie sont d'une immense valeur pour les études littéraire et restent source d'inspiration pour les poètes $\mathrm{du}$ $\mathrm{XX}^{e}$ siècle ainsi que pour les contemporains. Dans les textes théoriques, ou dans la métapoésie de certains poètes contemporains, on peut voir de manière claire la contribution des notions et des concepts de Valéry. Dans les métapoèmes d'Andrée Chedid ou lors des occasions où la poète parle de la poésie, on remarque l'important héritage de la critique de Valéry dans ses conceptions de poésie, du langage et de la création poétique.

Pour Valéry, " tout véritable poète est nécessairement un critique de premier ordre» (VALÉRY, 2002, p. 686). Le poète doit toujours avoir une réflexion critique sur sa propre création, sur tout le processus de construction d'un poème. Dans son essai intitulé Poésie et pensée abstraite il défend, entre autres, la notion d'un poète critique, capable d'un « raisonnement abstrait "sur le langage. Par rapport à la forme de son essai et à son écriture, il met en pratique ce qu'il défend : une critique de poète, avec toutes ses particularités et toutes ses différences par rapport à une critique faite par les théoriciens. Mireille Calle-Gruber, dans le livre Histoire de la littérature française du $\mathrm{XX}^{\mathrm{e}}$ siècle ou les repentirs de la littérature, parle d'une " critique d'écrivain ", plus générale certes, mais concernant celle des poètes. Elle classe la critique littéraire du XX $\mathrm{X}^{\mathrm{e}}$ siècle en " trois formes de critique » :

L'une, journalistique et radio-télévisée, qui rend compte de l'actualité littéraire ; l'autre, universitaire, soucieuse de recul vis-à-vis de l'œuvre, de la prise en compte de l'histoire, de sa réception, des apports de la théorie. La troisième est une critique d'écrivain laquelle, lisant-écrivant avec l'autre, fait œuvre singulière (CALLE-GRUBER, 2001, p. 69 - italiques de l'auteure).

fragmentum, n. 51, Jan./Jun. 2018. 
Pour elle, la critique d'écrivain relève aussi de la création et surtout d'une " exigence d'écrire " (CALLE-GRUBER, 2001, p. 82). Elle naît du besoin d'écrire "avec l'autre ", de réagir sur le texte de l'autre tout en créant une " œuvre singulière " : " $[\ldots]$ dans l'exercice de lecture du texte de l'autre qu'est la critique, se déclare une écriture : elle ne préexiste pas ; elle est désarmée ; elle est appelée par la découverte de l'œuvre, c'est une co-naissance. Une dynamique de la création donne lieu au texte critique " (CALLE-GRUBER, 2001, p. 84). Dans ce sens, cette critique faite par les écrivains donne naissance à des textes avec des caractéristiques bien particulières soit par rapport au fond, c'est-à-dire, le regard porté sur l'œuvre et la manière de l'analyser, soit par rapport à la forme, c'est-à-dire, les choix stylistiques dans l'écriture du texte :

Loin des certitudes des systèmes, si subtils soient-ils, et des terminologies tout employées à désigner, décrire, cataloguer, la critique d'écrivain entend au contraire l'abandon des principes qui garantissent notre culture ; elle exige d'aller à la limite, de rompre la propension totalisante et totalitaire des concepts, laquelle fonde l'histoire (CALLE-GRUBER, 2001, p. 83).

Ce que Mireille Calle-Gruber définit comme une " critique singulière » est celle dont la manière de construire des concepts et des définitions est différente de celle des auteurs purement théoriques. La critique d'écrivain étonne souvent le lecteur par la justesse de ses propos mais aussi par la beauté de son écriture car, dans la mesure où elle est également création et donne aussi un plaisir esthétique à celui qui la lit. Cette critique est ainsi peuplée de figures de style propres aux textes littéraires, comme des associations, des comparaisons, des métaphores ou même des oxymores. William Marx (2005), dans son livre sur Valéry, Eliot et la critique moderne, utilise l'expression "poète-critique " pour parler de ces deux poètes. Il les insère également dans une "tradition poétique française " (MARX, 2005, p. 18) inaugurée par Baudelaire et souligne les différences entre leurs œuvres critiques et celles des auteurs plus académiques :

\footnotetext{
Eparse, mobile, multiforme, si l'œuvre critique des deux poètes n'a pas la solidité ou la carrure de contributions plus académiques, elle reflète d'autant mieux, et qui plus est avec un brio particulier, la diversité des enjeux autour desquels se constitua la critique formaliste (MARX, 2005, p. 17).
}

Selon William Marx, la critique de Valéry est d'une grande originalité 
et importance au sein de la critique formaliste qui fait son apparition au $\mathrm{XX}^{\mathrm{e}}$ siècle. Pour lui, Valéry et T. S. Eliot auraient inventé une "nouvelle méthode » en s'opposant aux critiques étiologique et impressionniste de l'époque :

La critique étiologique aborde l'œuvre par le haut (ses sources, sa genèse, etc.), la critique impressionniste l'approche par en bas (les impressions sur le lecteur, l'effet moral, etc.), et entre les deux se dessine insensiblement une figure ou une forme encore intacte : celle de l'œuvre elle-même, ou en ellemême (MARX, 2005, p. 37).

William Marx souligne encore l'originalité de la critique de Valéry en l'opposant à la critique universitaire de l'époque :

\begin{abstract}
Autant la critique universitaire cherchait à accumuler le plus grand nombre possible de documents et accordait aux sources extra-littéraires (correspondances, papiers personnels, etc.) une importance prééminente dans son travail, autant la critique valéryenne veut limiter au maximum son objet jusqu'à ne plus conserver dans son champ d'observation que l'œuvre même, voire moins que l'œuvre : la simple idée de l'œuvre (MARX, 2005, p. 25).
\end{abstract}

Les théoriciens ne cessent donc pas de montrer toute la particularité, mais aussi les atouts de cette critique " multiforme » et "singulière " propre aux poètes. Les textes critiques de Baudelaire, les lettres de Rimbaud ou les essais de Mallarmé et de Valéry sont des textes qui procurent, justement, un plaisir autre que les textes appartenant à une « critique universitaire ». À part les caractéristiques formelles propres à ce type de critique, sur le plan des idées et des contenus, ils opèrent un mélange entre ressenti personnel, c'està-dire, l'observation de leurs propres expériences comme poètes, et réflexion critique sur l'écriture. Ainsi, la description d'une ouvre ou d'un concept n'est jamais complètement impersonnelle.

Dans un essai publié dans Curiosités esthétiques, Baudelaire défend une critique personnelle, "partiale " et " passionnée " contre une critique purement descriptive, «froide et algébrique». Il défend également la critique comme création - " amusante et poétique » comme un texte littéraire :

Je crois sincèrement que la meilleure critique est celle qui est amusante et poétique ; non pas celle-ci, froide et algébrique, qui, sous prétexte de tout expliquer, n'a ni haine ni amour, et se dépouille volontairement de toute espèce de tempérament [...]. Pour être juste, c'est-à-dire pour avoir sa raison d'être, la critique doit être partiale, passionnée, politique, c'est-à-dire faite à 
un point de vue exclusif, mais au point de vue qui ouvre le plus d'horizons (BAUDELAIRE, 1980, p. 641).

On constate que sa conception d'une critique idéale est finalement proche de celle de la critique d'écrivain définie par Mireille Calle-Gruber. Baudelaire résume très bien les particularités de cette critique et anticipe les revendications de Valéry. Pour Valéry, "il n’est pas de théorie qui ne soit un fragment, soigneusement préparé, de quelque autobiographie " (VALÉRY, 2002, p. 666). Ainsi, dans Poésie et pensée abstraite, il remplit son texte de descriptions de ce qu'il observe en lui-même, des " fragments de son autobiographie " de poète : "j'ai donc observé en moi-même tels états que je puis bien appeler Poétiques, puisque quelques-uns d'entre eux se sont finalement achevés en poèmes " (VALÉRY, 2002, p. 665). Une autre particularité de cette critique d'écrivain, rencontrée dans l'essai de Valéry, mais aussi dans les lettres de Mallarmé, est la présence des explications sur les propres procédés utilisés dans la critique. Mallarmé explique ainsi, au destinataire de sa lettre, ce qu'il recherche comme critique : « [...] si j’ai un principe quelconque en Critique, c'est qu'il faut, avant tout, rechercher la pureté des genres » (MALLARMÉ, 1995, p. 555).

Et Valéry expose ses procédés dans ses analyses critiques :

Quant à moi, j'ai la manie étrange et dangereuse de vouloir, en toute matière, commencer par le commencement (c'est-à-dire, par mon commencement individuel) [...]. En toute question, et avant tout examen sur le fond, je regarde au langage ; j'ai coutume de procéder à la mode des chirurgiens qui purifient d'abord leurs mains et préparent leur champ opératoire. C'est ce que j'appelle le nettoyage de la situation verbale (VALÉRY, 2002, p. 661 italiques de l'auteur).

On rencontre des explications sur la propre critique qu'ils sont en train de réaliser, une sorte de théorisation de la critique. C'est un travail de réflexion dans la propre réflexion qui ne fait que prouver le besoin des poètes critiques de réfléchir constamment sur leur écriture, qu’elle soit littéraire ou même théorique.

Mais revenant sur le fait que la critique d'écrivain est aussi création, ou possède une grande partie de création, on constate que chaque auteur, chaque ouvrage ont leurs caractéristiques propres. On y trouve, certes, des aspects communs, mais chaque écrivain saura mettre sa part de personnalité dans sa critique. On peut noter que malgré les variations d'écriture, il y a une 
marque significative qui apparaît dans nombreux textes critiques des auteurs étudiés lors de cette recherche : le dialogue que ces textes établissent avec le lecteur. Comme conséquence de toutes les particularités citées ci-dessus, la critique d'écrivain établit avec son lecteur une relation très particulière, elle dialogue directement avec son lecteur et le rend actif et coopératif (ECO, 2008). Il existe la création d'une certaine complicité entre les deux assez proche de celle établie dans les textes littéraires.

Toutes ces spécificités font que la critique des poètes soit si importante pour comprendre la poésie moderne et contemporaine et qu'elle ait gagné tant d'espace et de reconnaissance dans les études littéraires. Jean-Michel Maulpoix dénonce, dans l'introduction de son livre sur le « lyrisme critique ", la faillite de la critique de " l'Université » et de celle des " médias " face à l'écriture de "l'extrême contemporain » et défend que seul un « lyrisme critique » puisse la saisir :

Face à ce déficit d'une écriture critique qui serait capable d'appréhender " l'extrême contemporain " et de le confronter à sa mémoire aussi bien qu'à son amnésie, je réaffirme la nécessité du lyrisme critique, c'est-à-dire du geste réflexif inhérent à l'écriture même, telle qu'elle invente, analyse et réfracte. La critique trouve refuge là où elle prend naissance : dans l'incessante relecture que fait l'écrivain de ce texte qu'il devient, dans cette surveillance où il tient ses abandons, ses impulsions ou ses impuissances (MAULPOIX, 2004, p. 13).

Et ce que Maulpoix comprend comme "lyrisme critique " est finalement la critique faite par des poètes, sous la forme théorique ou poétique :

J'entends aussi bien par " lyrisme critique " l'état auquel la poésie parvient, quand elle a pris conscience que l'heure n'est plus à l'invention de nouvelles formes, mais refuse de céder à la tentation du bricolage postmoderne, pour se rendre suprêmement attentive aux éclats de sa voix et mesurer objectivement les forces qui la mobilisent ou l'étranglent (MAULPOIX, 2004, p. 13-14).

A partir de la définition de poète critique de Valéry ou de celle de "lyrisme critique " de Maulpoix, on peut constater que, dans les deux cas, la notion de poète critique concernerait tous les poètes réfléchissant sur la poésie, la création du poème et sur leurs processus d'écriture, ce qui englobe alors les textes théoriques, mais aussi la métapoésie. 


\section{Les poètes critiques contemporains : entre théorie et métapoésie}

La critique littéraire française est fortement marquée par la présence de textes théoriques écrits par des poètes. Mireille Calle-Gruber (2001) et Jean-Michel Maulpoix (2004) ont constaté la dimension de cette critique singulière dans la scène littéraire contemporaine. Cela est notamment due au fait que, comme l'affirme Maulpoix, elle serait la seule à être capable de comprendre les nuances de la poésie contemporaine, mais aussi par la quantité significative de poètes contemporains qui se sont aventurés dans des textes critiques ou qui ont, dans leurs poétiques, mélangé critique et poésie.

À part cette singularité de la critique d'écrivain, il faut également noter, comme le fait Mireille Calle-Gruber et d'autres théoriciens ${ }^{4}$, que le $\mathrm{XX}^{\mathrm{e}}$ siècle et la poésie contemporaine ont été marqués par un grand nombre de textes littéraires interrogeant l'écriture elle-même. Les réflexions critiques sont ainsi présentes non seulement dans des essais ou des ouvrages théoriques, mais aussi dans la propre littérature ce qui explique la présence d'une grande quantité de métapoèmes dans la poésie française contemporaine. La conception de poète critique concerne les poètes ayant produit des textes critiques et ceux qui ont produit des poèmes contenant des réflexions métapoétiques :

\footnotetext{
Notons encore que ce siècle, passionné de formes et de techniques, qui ne renie plus le travail de l'œuvre mais au contraire rend celle-ci à la scène de l'opéra (ouvroir, ouvrage, théâtre, transfigurations), ce $\mathrm{XX}^{\mathrm{e}}$ siècle aura cherché à interroger de toutes les façons possibles le secret du travail d'écrire - la fabrique, le chiffre, l'énigme, le schibboleth (CALLE-GRUBER, 2001, p. 85).
}

Daniel Briolet confirme plusieurs fois dans son livre dédié à la poésie française $\mathrm{du} X \mathrm{XX}^{\mathrm{e}}$ siècle, une forte présence critique dans la poésie contemporaine, notamment celle de la période des années 1960 aux années 1990 : «Ce souci d'articuler sans cesse création poétique et réflexion critique semble caractériser de façon prépondérante la poésie des trois décennies écoulées de 1960 à 1990 »(BRIOLET, 1995, p. 139).

Philippe Delaveau parle de l'" extrême lucidité " (DELAVEAU, 1988, p. 7) comme la caractéristique la plus facilement remarquable de la poésie

${ }^{4}$ Cf. DELAVEAU (1988); BLANCQUART (1996); BRINDEAU (1973); LEUWERS (2001) et BRIOLET (1995). 
moderne et contemporaine. Il signale cette tendance d'unir dans la même démarche, poésie et poétique, laquelle est poussée plus avant par des poètes contemporains comme Michel Deguy et Jean-Claude Renard. Leurs œuvres sont marquées par une profonde réflexion sur ce que Deguy appelle "le langage du langage »(DELAVEAU, 1988, p. 19). Ces deux poètes ainsi que d'autres contemporains comme Lorand Gaspar ou Jean-Michel Maulpoix ont, également écrit d'importants textes critiques en prose sur la création poétique et le langage.

Par ailleurs, Daniel Briolet constate, dans ses études, l'existence d'une métapoésie significative de cette période, mais aussi d'une critique nouvelle faite par des poètes. Cette critique est, pour lui, l'héritage de la critique des poètes du XIX et $\mathrm{XX}^{\mathrm{e}}$ siècle et le champ de "productions fort différentes " associant de manière singulière "création poétique et réflexion critique " (BRIOLET, 1995, p. 139):

Le début des années soixante a vu se multiplier ce qu'il est convenu d'appeler "les critiques nouvelles". Leur prolifération depuis cette époque témoigne sans ambiguïté de cette tension vers l'infini du sens qui suscite, comme on vient de voir, toute œuvre du langage. Elle provient pour une part de l'immense effort de réflexion théorique mené tout au long du siècle par les poètes eux-mêmes. Beaucoup appelaient de leurs vœux une véritable révolution critique. Tous, ou presque tous, ont ouvert la voie aux analyses critiques les plus fécondes (BRIOLET, 1995, p. 178).

Carmen Boustani (2003) et Mireille Calle-Gruber (2001) voient aussi, dans la diversité des formes et le mélange des genres, la richesse de la critique faite par des poètes contemporains. Boustani affirme que des « textes hybrides combinant récit, journal intime, épistolaire, poésie et réflexion théorique " (BOUSTANI, 2003, p. 16) font la spécificité et la "modernité " de textes produits par des poètes français de ces dernières décennies. Selon Calle-Gruber, « les nouveautés en création et critique littéraires » (CALLEGRUBER, 2001, p. 71) en France viennent de la poésie, notamment des poètes critiques. Parmi ces poètes, elle remarque le rôle fondamental du poète Michel Deguy, auteur d'ouvrages critiques emblématiques comme La poésie n'est pas seule - Court traité de poétique, de 1987 et La raison poétique, de 2000 .

La production des poètes critiques contemporains compte ainsi des textes théoriques - classés comme des essais, des textes hybrides - parfois classés comme de la prose poétique - et des métapoèmes. Dans le cadre de la 
recherche qui a abouti à cette thèse, l'étude de certains poètes et de certains ouvrages s'est révélée importante pour enrichir et compléter la compréhension d'une poétique contemporaine tournée vers elle-même. Devant l'abondance, la diversité et la richesse de cette poésie critique contemporaine de langue française, il est très difficile de tracer un panorama ou même de faire un simple état de lieu du contexte poétique des sept dernières décennies. Serge Brindeau, dans le livre La poésie contemporaine de langue française depuis 1945, publié en 1973, cite mille poètes, parmi lesquels ceux qui ont écrit de la métapoésie seraient difficilement dénombrables.

Parmi les essais critiques, nous soulignons l'importance des livres Approche de la parole de Lorand Gaspar, Notes sur la poésie de Renard et Pour un lyrisme critique de Maulpoix. En ce qui concerne la métapoésie, certains métapoèmes de Jacques Dupin, d'André du Bouchet, de Philippe Jaccottet, de Eugène Guillevic et de Jean Tardieu ont retenu notre attention. Renard et Gaspar ont éprouvé le besoin de réfléchir sur la poésie, le processus créateur et leurs propres poétiques. Ainsi que d'autres poètes de leur génération, ils ont écrit des métapoèmes, mais leurs ouvrages en prose restent la partie la plus expressive de leur critique. Ces ouvrages illustrent toute la singularité que les théoriciens accordent à la critique des poètes. Ce sont des « textes hybrides" (BOUSTANI, 2003, p. 16) où se mélangent la théorie littéraire, la philosophie et, selon notre interprétation, la littérature. Dans cette étude nous adoptons la conception de "critique écrivain " de Mireille Calle-Gruber (2001) qui voit dans la critique des poètes une part de création plus significative que dans celle des théoriciens de la littérature. Les ouvrages de Gaspar et de Renard, cités ci-dessus, ont donc une écriture proche de celle des ouvrages critiques de Valéry et de son contemporain Pierre Reverdy.

Parmi les ouvrages de ces deux poètes, celui de Lorand Gaspar (1978) reste le plus singulier ou hybride. Dans la quatrième de couverture de l'édition de 1978, l'éditeur termine son texte par une affirmation qui confirme toute notre difficulté de le classer dans un genre précis, et sa réflexion devient peu à peu poème. Lorand Gaspar est né en Hongrie en 1925. Déporté lors de la Seconde Guerre, il trouve refuge en France où il fait des études de médecine. Il vit longtemps à Jérusalem et le désert est une constante dans son œuvre. Se tournant vers sa critique dans le livre Approche de la parole (1978) on rencontre parmi ses propos, maintes ressemblances avec ceux contenus dans la métapoésie.

Il aborde ainsi la relation entre poésie et langage, parlant du travail du 
poète dans la transformation du langage - tel que les combinaisons de mots ou leur « remodelage »:

Tout se passe comme s'il y avait dans la vie de l'homme qui demandait à se manifester, à être communiqué et ne le pouvait qu'en " jouant " avec le langage, en le défaisant et en le remodelant, en le décapant, en le creusant, en y descellant des énergies, des rapports, des liaisons méconnues, oubliées, recouvert de quelque croûte d'oxydation, enserrés dans le carcan d'un processus fibreux. Comme si cette chose inconnue ou ensevelie en venant à la langue y rencontrait à l'abord une insuffisance, une inadéquation. Comme si elle ne supportait aucune formulation arrêtée, aucune fermeture. Pour arriver tout de même à la parole, ou du moins le tenter, il faut alors réapprendre à parler (GASPAR, 1978, p. 51).

Le langage poétique est pour Gaspar un lieu " où apparaît ou s'effondre le monde ", de la même façon qu'il est, pour Chedid, un lieu où "se greffe la totalité »(CHEDID, 1999, p. 117) de la vie : "Sans cesse la langue défait et refait le jeu de son tissage de signes. Elle est grève mouvante où apparaît et s'effondre le monde, elle est ardeur à vivre en corrosion de ses limites, aux approches de ce qui la consume " (GASPAR, 1978, p. 32-33).

Dans Approche de la parole, Gaspar suggère aussi des propos proches de ceux de Chedid par rapport aux questionnements auxquels la poésie n'arrive pas à répondre et dont l'objectif est justement la "non-réponse " (CHEDID, 2006, p. 88) ou encore sur l'impossibilité de définir la poésie :

Le poème n'est pas une réponse à une interrogation de l'homme ou du monde. Il ne fait que creuser, aggraver le questionnement. Le moment le plus exigeant de la poésie est peut-être celui où le mouvement (il faudrait dire la trame énergétique) de la question est tel - par sa radicalité, sa nudité, sa qualité irréparable - qu'aucune réponse n'est attendue ; plutôt, toutes révèlent leur silence (GASPAR, 1978, p. 35).

Il justifie encore dans un autre extrait le principe même de la métapoésie et suggère, en même temps, l'impossibilité de décrire la poésie :

Ce que les poètes ont à dire de la poésie, c'est encore de la poésie [...]. Si réponse il y a aux questions que pose l'existence du phénomène poétique, elle ne peut être que dans la chair vive de cette venue, et seulement par une sorte de négation dans les analyses et les explications (GASPAR, 1978, p. $52-53)$. 
Le livre Notes sur la poésie de Jean-Claude Renard est divisé en deux parties, dont la première porte le titre de Langage, poésie et réalité. Les cinq premiers chapitres y sont respectivement intitulés : Le poète et le langage, Genèse du poème, Langage et poésie, Le langage poétique et Les mots, ils possèdent, dans leurs contenus, certains points communs avec la critique de Paul Valéry et notamment avec celle d'Andrée Chedid. Renard y aborde entre autres le pouvoir du langage poétique de "donner droit au revers des images » (CHEDID, 1987, p. 136) ou les multiples possibilités qu'il offre, ainsi que les spécificités du langage poétique telles que les associations de mots - préconisées par Valéry et par Chedid :

\footnotetext{
Le langage poétique a la propriété paradoxale d'être à la fois l'endroit et l'envers du langage, de poser ensemble et en même temps une affirmation et une négation, ce qui est dit et ce qui n'est pas dit ou ne peut pas être dit, de se présenter simultanément comme écriture et comme non-écriture, comme science du langage et comme œuvre du langage, comme fragment et comme discours : fragment chargé d'une possibilité de sens qui le continue, - discours chargé de suspensions de sens qui le rompent.

Peut-être est-ce cette même faculté qui lui donne le pouvoir d'associer en lui ce qui ne paraît pas l'être dans la réalité $[\ldots]$ - et de devenir ainsi le lieu où "les contraires s'accordent ", où " de toutes choses naît l'un et l'un de toutes choses ", et où peuvent apparaître à la fois l'avers et le revers d'un même mot, d'un même sens, d'une même réalité (RENARD, 1970, p. 29).
}

Parmi les auteurs étudiés, Jean-Michel Maulpoix est le seul qui n’appartient pas à la génération de Chedid, il est né en 1952 et publie l'ouvrage analysé dans cette étude - Pour un lyrisme critique - en 2009. Jean-Michel Maulpoix a écrit de la poésie et des textes critiques. Parmi ses ouvrages, les textes critiques restent les plus reconnus en France. Le livre Pour un lyrisme critique fait partie d'une série de textes critiques sur la poésie composée de quatre autres titres : La voix d'Orphée (1989), Du lyrisme (2000), Le poète perplexe (2002) et Adieux au poème (2005). Dans ce livre, il développe de manière plus approfondie la notion de lyrisme critique qui apparaît déjà dans les ouvrages précédents. Cette notion est au centre de la métapoésie contemporaine, étant la justification même de cette poésie critique si présente dans la scène littéraire française contemporaine.

Par ailleurs, l'ouvrage de Maulpoix est un bon exemple de la critique singulière produite par les poètes, car, comme Valéry, en même temps que le poète la pratique, il tient à la justifier par des explications significatives sur les spécificités de ses analyses et de l'écriture de son texte. Ainsi commence- 
t-il l'avant-propos en avertissant son lecteur du caractère et de la tonalité de sa critique : "Le lecteur chercherait en vain dans les pages qui suivent l'affirmation d'une doctrine. En dépit de son titre, ce livre n'est pas un manifeste. Il marque une simple étape dans une réflexion toujours en cours sur le lyrisme " (MAULPOIX, 2004, p. 9). On revient à la question de l'opposition entre poésie et pensée abstraite reformulée par Maulpoix dans l'opposition entre lyrisme et critique :

- Qu'entendez-vous par lyrisme critique ? La question me fut souvent posée. En effet, attacher l'adjectif " critique " au mot " lyrisme " ne va pas de soi. Celui-ci n'évoque-t-il pas un emportement, une parole inspirée que conduit l'enthousiasme, une ivresse verbale peu propice à la réflexibilité et qui s'enchante des capacités sonores du langage au détriment du sens des mots? Je voudrais à nouveau montrer ici que le lyrisme n'est pas réductible à cette idée simpliste d'un flux verbal peu ou mal contrôlé, non plus qu'à une quelconque effusion de sentiments. [...] Il invite à interroger l'ensemble des forces que l'écriture poétique met en jeu et des résistances qu'elle rencontre. Il constitue aussi bien un objet critique qu'un lieu critique, et il engage par ailleurs quiconque lui reconnaît une valeur à un certain type d'écriture critique (MAULPOIX, 2004, p. 9-10).

Il met en jeu, comme le font Valéry et Chedid, deux forces longtemps opposées en poésie, celle de l'inspiration et celle du travail sur le langage. Et pour lui comme pour Valéry, le travail sur le langage est le propre de la poésie, celui-ci est indissociable d'une réflexion critique. Quant à Maulpoix, il considère que le lyrisme critique est le "geste réflexif inhérent à l'écriture même " (MAULPOIX, 2004, p. 13), c'est l'état où la poésie se rend "suprêmement attentive aux éclats de sa voix" (MAULPOIX, 2004, p. 13-14) et arrive à " mesurer objectivement les forces qui la mobilisent " (MAULPOIX, 2004, p. 14).

En ce qui concerne la métapoésie des poètes contemporains à Andrée Chedid, nous avons retenu quelques exemples qui nous semblent significatifs de cette génération très marquée par une vision critique de la poésie et de sa propre poétique. Parmi ces ouvrages, il nous a paru important de souligner certains métapoèmes d'André du Bouchet, de Philippe Jaccottet, de Jean Tardieu, de Jacques Dupin et de Guillevic. Ces poètes ont laissé de leurs empreintes la poésie contemporaine de langue française. Par leur poésie, ils démontrent la variété et la richesse de cette scène. Et, s'ils s'éloignent les uns des autres au niveau de l'écriture, ils se rejoignent sur certaines thématiques, comme celle de la métapoésie. 
Jean Tardieu publie dans le livre Margeries (2009) une série de poèmes écrits entre 1924 et 1925 intitulée Le langage en question - musique de scène pour une thèse, dont le sujet est le langage. Dans cet ensemble, l'auteur aborde la relation entre langage et poésie et, dans le métapoème intitulé L'écran-langage, il justifie le principe même de la métapoésie, annonçant dès le début du métapoème qu'il écrit un poème dont le sujet est le langage - le même utilisé pour écrire le poème. On remarque donc un mouvement circulaire ou l'image de miroirs multiples - du langage qui regarde le langage, ou du langage qui se sert du langage pour s'analyser :

Je m’apprête à écrire un poème. Sur quel sujet ? Sur le langage.

Cette bizarre étude, cette entreprise audacieuse demande cependant que je m'exprime

en un langage !

Et voici où paraitra ce phénomène, la découverte :

le langage scrutera le langage

Or, pour indiquer cette simple idée :

"Mon langage scrutera le langage»,

il est bien évident que j'ai utilisé... quoi ? Mais oui :

le langage!

De sorte que nous obtenons ce résultat :

mon langage regarde que le langage regarde le langage!

Encore tout ne se termine-t-il pas ici, car c'est le langage qui vient de me servir lorsque je dis : "Mon langage... ", et ainsi de suite, vous comprenez : le langage du langage du langage du langage du langage du langage du langage

langage

langage

lang-âge

langue - âge

lent gage

l'an - gage

l'engage

langage langage langage langage langage langage...

[...] (TARDIEU, 2009, p. 60-61).

Nous voyons dans ce poème, le principe de la métapoésie - avec un regard autoréflexif et le constant questionnement sur elle-même - le langage 
qui parle du langage, comme la poésie qui analyse la poésie. Hackett, dans l'étude intitulée Panorama de la poésie française contemporaine, souligne le fait que «l'œuvre de Jean Tardieu est parsemée de réflexions sur le langage " (HACKET apud DELAVEAU, 1988, p. 20) et cite le poème L'écran-langage comme un des exemples les plus illustratifs de sa métapoésie. On remarque que l'écriture de Tardieu a un trait d'humour et une certaine manière de jouer avec les mots, sur leur sens et sur leur forme, qui transparait dans ce métapoème. Dans ce sens, Hackett (1988, p. 13-23) rapproche l'écriture métapoétique de Tardieu de celle de Raimond Queneau, on constate une métapoésie qui diffère de celle de Chedid et qui vient confirmer la diversité de la production des poètes contemporains de cette génération.

Un autre poète qui a attiré notre attention en lisant la métapoésie est Jacques Dupin dont les métapoèmes les plus significatifs sont publiés dans le recueil Echancré (1991). Ce recueil possède une séquence de métapoèmes qui définissent l'écriture poétique et, à travers elle, ses conceptions de poésie et sa propre poétique. Dans la plupart de ces métapoèmes, l'auteur utilise le verbe " écrire " à l'infinitif dans ce que nous avons perçu comme une sorte d'auto réaffirmation da sa propre démarche poétique. La répétition de ce verbe à l'infinitif, peut également suggérer des conseils ou les nuances d'une recette d'écriture poétique livrée par le poète à chaque métapoème :

Ecrire en se gardant du spéculaire, du simulacre, de la déflagration, du glissement... autour des yeux, au fond de l'œil, hors de portée du regard... écrire étant la traversée du souffle, l'impossible traversée... étant l'impossible... (DUPIN, 2009, p. 134).

Ce poème évoque un élément fréquemment utilisé dans la métapoésie d'Andrée Chedid - le "souffle ». Le souffle comme inspiration - don fait au poète - ou comme marque du rythme - respiration, cadence - références présentes dans quelques métapoèmes de l'auteure 5 . Le premier métapoème de cette séquence présente également une autre image récurrente dans la métapoésie de Chedid - le verbe " creuser " comme démarche ou caractéristique de l'écriture poétique :

Ecrire les yeux fermés. écrire la ligne de crête. écrire le fond de la mer...

creuser plus profond que le vagissement du nouveau-né, que le cri de la chasseresse, la plainte du supplicié... que l'enchevêtrement des racines, que

${ }^{5}$ Cf. CHEDID, Andrée. Territoires du souffle. Paris: Flammarion, 1999. 
l'exténuation des lanières de la terreur...

écrire sans recul, dans le noir. Dans la doublure, dans la duplicité, du noir... (CHEDID, 1999, p. 123).

Un autre élément de la poétique qui est abordé dans la métapoésie de Dupin est le rythme. Il y fait allusion dans un des métapoèmes du recueil où apparait dans un même temps, l'association d'images contraires suggérant ainsi l'ampleur de la poésie : " écrire : une écoute - une surdité, une absurdité - écrire pour atteindre le silence, jouir de la musique de la langue, extraire le silence du rythme et des syncopes de la langue " (CHEDID, 1999, p. 124).

De plus, certains métapoèmes de Dupin suggèrent la quête sans fin, le « cheminement " qui est l'écriture poétique ou encore le pouvoir de cette dernière sur le poète :

Ecrire au fond du trou, écrire sur le fil, en disloquant, en moissonnant, en délivrant l'espace du vide vivant...

les moignons repoussent, le talon froidit, l'herbe est en fleurs sur l'autre versant... on ne finira jamais d'appeler le mot qui tombe, ni d'aligner la lettre qui jaillit d'une fissure de la pierre...

écrire sous la torture, dans la torpeur - ou ne pas écrire - et mourir d'écrire de n'être pas mort... (DUPIN, 2009, p. 144).

L'écriture de Dupin impose une expérience de l'abrupt et de l'écart, et exprime une négativité plus forte. Dans ce sens, elle est différente de celle de Chedid ou encore de celle de Tardieu même si, par rapport aux concepts exposés ou aux images utilisées, on peut toujours établir des correspondances entre leurs œuvres métapoétiques. André du Bouchet est aussi un des poètes dont l'œuvre métapoétique est importante et dont l'écriture poétique est fort différente de celle de Chedid. Les livres Dans la chaleur vacante (1961) et Ici en deux (1986) présentent des métapoèmes représentatifs de la métapoésie de l'auteur. La séquence de poèmes intitulée Image parvenue à son terme (DU BOUCHET, 1991, p. 109-115), du livre Dans la chaleur vacante, possède des extraits qui démontrent la complexité de l'écriture de Du Bouchet : 
banale que chacun de plein droit se l'approprie, comme si, à même l'obstacle qui un instant a pu nous en retrancher, l'élément rare - montagne ou évidence - de lui-même se déplaçait jusqu'à nous : que, poésie, rien du coup ne la distingue d'une réalité dont elle continue de tirer, sans en conserver de trace toujours reconnaissable, le pouvoir rudimentaire qui aveuglément nous a engagés.

\section{$[\ldots]$}

C'est encore ce souffle - perdu pour respirer, et perdu - dans l'instant même où, d'une parole que le poème a, semble-t-il, arrêtée, il nous arrivera d'induire que, notre fin, nous l'avons laissées derrière nous (DU BOUCHET, 1991, p. 111).

Dans le livre Ici en deux, le chapitre intitulé Notes sur la traduction (DU BOUCHET, 2011, p. 91-138) présente des métapoèmes qui traitent du langage - de la " parole » et des " mots » :

peut-être, par le travers de la parole, elle aussi les choses auront-elle pu voir (DU BOUCHET, 2011, p. 108).

quant au sens, autant de ciel ouvert que de de terre occupée. Mais la terre est ouverte (DU BOUCHET, 2011, p. 109).

mots comme signes de la ponctuation prononçable subitement - et sur lesquels, ouvrant alors à autre chose, l'esprit qui portera sur l'intervalle renouvelé, demeure en suspens (DU BOUCHET, 2011, p. 112).

Dans ces extraits, on rencontre la suggestion du pouvoir de la parole et de l'ouverture de sens, mais aussi l'ouverture d'esprit vers de multiples horizons que la poésie favorise - des caractéristiques que les poètes critiques attribuent au langage poétique. En outre, on rencontre dans d'autres extraits, l'allusion à une langue poétique qui serait la traduction de « la langue de l'autre qui est en soi " - comme l'a fait Arthur Rimbaud avec la célèbre formule " je est un autre " : " autre langue. Ou autre chose. Déjà la langue de l'autre qui est en soi " (DU BOUCHET, 2011, p. 112).

$\mathrm{Ou}$ encore, de cette même séquence, le poète fait référence aux associations de mots, un thème cher à Chedid et à d'autres poètes critiques : 
mots - et d'un mot à un autre, comme ils s'inscriront, accentuant le vide préparatoire à autre vie ou à autre mot - emportant les mots (CHEDID, 1999, p. 123).

un mot : morceau d'espace. Un mot : sur une attente du sens procuré par le mot à venir - qui complétera.

la cassure - sa fierté (CHEDID, 1999, p. 123).

Ces Notes sur la traduction sont, finalement, pour nous, davantage des notes sur le langage poétique que sur la connotation usuelle du mot " traduction ". Selon Michel Colot, dans la préface de l'édition Gallimard de 2011, il faut penser ce titre comme " un double sens " : la traduction d'autres langues, mais aussi celle opérée par la poésie :

C'est dire que la problématique de la traduction [...] doit être comprise en un double sens : le passage d'une langue à une autre s'y avère inséparable de la poésie comme tentative pour «traduire " ce qui, du monde, semble se soustraire à la nomination et n'a pas encore été exprimé (DU BOUCHET, 2011, p. 12).

Maulpoix considère le poète Philippe Jaccottet comme représentatif de la génération de Chedid, cette même génération très consciente et critique de la poésie ou de sa propre écriture. Pour lui, Jaccottet incarne la figure du "poète soucieux de prendre une juste mesure de ce qu'il est et de ce qu'il peut accomplir " (MAULPOIX, 2002, p. 288) en tant que poète et souligne l'importance donnée à la définition de la tâche du poète dans le texte "Le travail du poète ", du recueil L'ignorant (JACCOTTET, 2011, p. 29-59) :

L'ouvrage d'un regard d'heure en heure affaibli

n'est pas plus de rêver que de former des pleurs,

mais de veiller comme un berger et d'appeler

tout ce qui risque de se perdre s'il s'endort (JACCOTTET, 2011, p. 36).

Le premier poème du recueil Leçons (JACCOTTET, 2011, p. 215240) reprend l'image du poète et fait l'opposition entre ce que Maulpoix définit comme le "poète précoce " (MAULPOIX, 2002, p. 294)- ou le jeune poète qui a été Jaccottet - et le " poète tardif» (MAULPOIX, 2002, 
p. 294) qu'il est devenu :

Autrefois,

moi l'effrayé, l'ignorant, vivant à peine,

me couvrant d'images les yeux,

j'ai prétendu guider mourants et morts.

Moi, poète abrité,

Epargné, souffrant à peine,

aller tracer des routes jusque-là !

A présent, lampe soufflée,

main plus errante, qui tremble,

je recommence lentement dans l'air (MAULPOIX, 2002, p. 294).

Dans le recueil Chants d'en bas (JACCOTTET, 2011, p. 241-257), une séquence de poèmes intitulée Parler, compare le verbe de son titre à la poésie :

Parler pourtant est une autre chose, quelquefois,

que se couvrir d'un bouclier d'air ou de paille...

Quelquefois c'est comme en avril, aux premières tiédeurs, quand chaque arbre se change en source, quand la nuit

semble ruisseler de voix comme une grotte

(à croire qu'il y a mieux à faire dans l'obscurité des frais feuillages que dormir),

cela monte de vous comme une sorte de bonheur,

comme s'il fallait, qu'il fallût dépenser

un excès de vigueur, et rendre largement à l'air

l'ivresse d'avoir bu au verre fragile de l'aube.

Parler ainsi, ce qui eut nom chanter jadis

et que l'on ose à peine maintenant,

est-ce mensonge, illusion ? Pourtant, c'est par les yeux ouverts

que se nourrit cette parole, comme l'arbre par ses feuilles.

Tout ce qu'on voit,

tout ce qu'on aura vu depuis l'enfance, 
précipité au fond de nous, brassé, peut-être déformé

ou bientôt oublié

[...]

tout cela qui remonte en paroles, tellement

allégé, affiné qu’on imagine

à sa suite guéer même la mort... (JACCOTTET, 2011, p. 251-252).

L'image du " fond de nous " y apparaît et elle est proche de celle des métapoèmes de Chedid - comme synonyme d'âme ou de source où s'origine le poème. Il y a également l'allusion à la transformation de l'inspiration en poème - quand " tout cela remonte en paroles " - et au travail du poète qui " allège " et " affine " ce chant. Guillevic suggère, dans certains métapoèmes le rapprochement de la poésie avec la vie - le poème comme " reflet " du monde :

Dans le poème

On peut lire

Le monde comme il apparaît

Au premier regard.

[...] (GUILLEVIC, 2001, p. 178).

Le poème

Nous met au monde (GUILLEVIC, 2001, p. 291).

Ses métapoèmes évoquent aussi les caractères inépuisable et indéfinissable de la poésie :

Et si le poème

Etait une bougie

Qui se consumerait

Sans jamais s'épuiser ? (GUILLEVIC, 2001, p. 217).

On y retrouve également des images qui lient la poésie au «chant " 
et au « mystère " :

Le chant

Peut être silence.

Le silence peut exister

Pour qui chante,

Pour lui

Et pour tous,

Car il porte le chant

A travers les horizons (GUILLEVIC, 2001, p. 157).

Et finalement, comme Chedid, Guillevic nous livre, dans son art poétique, sa démarche comme poète :

Ce que je crois ne pas savoir,

Ce que je n'ai pas en mémoire,

C'est le plus souvent,

Ce que j'écris dans mes poèmes (GUILLEVIC, 2001, p. 172).

Un travail : créer

De la tension

Entre les mots,

Faire que chacun

En appelle un

Ou plusieurs autres [...] (GUILLEVIC, 2001, p. 280).

Les exemples de la métapoésie et de la critique de ces quelques poètes contemporains, nous démontre que même avec les différences d'écriture, dans leur métapoésie, d'une manière générale, les thèmes et les concepts se répètent au point que l'on arrive à y trouver des correspondances. 
Notre regard sur la métapoésie contemporaine, même si insuffisamment étendu, nous laisse voir, à travers ces correspondances, une certaine unité thématique. La communion (ou le dialogue ou le partage) d'idées ainsi que celle des perceptions de la poésie et de l'univers poétique nous permettent de trouver des liaisons et des rapprochements possibles entre les textes critiques de poètes de langue française, du XX' siècle à nos jours.

\section{Références}

BAUDELAIRE, Charles. Euvres complètes. Paris: Ed. Robert Laffont, 1980.

BLANCQUART, Marie-Claire. La poésie en France du surréalisme à nos jours. Paris: Ellipses, 1996.

BONNEFOY, Yves. Poèmes. Paris: Gallimard, 2001.

. L'alliance de la poésie et de la musique. Paris: Galilee, 2007.

. Entretiens sur la poésie. Paris: Mercure de France, 1991.

. L'inachevable. Paris: LGF, 2012.

BOUSTANI, Carmen. Aux frontières des deux genres, en hommage à Andrée Chedid. Paris: Karthala, 2003.

BRINDEAU, Serge. La poésie contemporaine de langue française depuis 1945. Paris: Ed. Saint-Germain-des-Prés, 1973.

BRIOLET, Daniel. Lire la poésie française du $\mathbf{X X}^{\mathrm{e}}$ siècle. Paris: Dunod, 1995.

BRUNEL, Pierre. Histoire de la littérature française. Tome 2. Paris: Editions Bordas, 1977. 
CALLE-GRUBER, Mireille. Histoire de la littérature française du $\mathbf{X X}^{\mathbf{e}}$ siècle ou Les repentirs de la littérature. Paris: Honoré Champion, 2001.

CHEDID, Andrée. Textes pour un poème (1949-1970). Paris: Flammarion, 1987.

. Territoires du souffle. Paris: Flammarion, 1999.

. Entre Nil et Seine. Entretiens avec Brigitte Kernel. Paris: Belfond, 2006.

DEGUY, Michel. La poésie n'est pas seule - court traité de poétique. Paris: Seuil, 1987.

La raison poétique. Paris: Editions Galilée, 2000.

DELAVEAU, Philippe. La poésie française au tournant des années 1980. Paris: Corti, 1988.

DU BOUCHET, André. Dans la chaleur vacante. Paris: Gallimard, 1991. . Ici en deux. Paris, Gallimard, 2011.

ECO, Umberto. Lector in fabula. Paris: LGF, 2008.

FRIEDRICH, Hugo. Structure de la poésie moderne. Paris: LGF, 1999.

GASPAR, Lorand. Approche de la parole. Paris: Gallimard, 1978.

GUILLEVIC, Eugène. Art poétique. Paris: Gallimard, 2001.

HACKETT, Cecil Arthur. «Panorama de la poésie française contemporaine ». In: DELAVEAU, Philippe (Dir.). La poésie française au tournant des 
années 1980. Paris: Corti, 1988, p. 13-23.

JACCOTTET, Philippe. L'encre serait de l'ombre. Paris: Gallimard, 2011.

LAGARDE, André; MICHARD, Laurent. La littérature du XIX ${ }^{\mathbf{e}}$ siècle. Paris: Editions Bordas, 1967.

LEUWERS, Daniel. Les lettres du voyant Rimbaud. Paris: Ellipses, 1998.

MALLARME, Stéphane. Igitur, Divagations, Un coup de dés. Paris: Gallimard, 2003.

. Correspondance complète suivi de Lettres sur la poésie. Paris: Gallimard, 1995.

MARX, William. Naissance de la critique moderne. La littérature selon Eliot et Valéry. Arras: Artois Presses Université, 2005.

MAULPOIX, Jean-Michel. La voix d'Orphée. Paris: José Corti, 1989.

. Du lyrisme. Paris: José Corti, 2000.

Le poète perplexe. Paris: José Corti, 2002.

. Pour un lyrisme critique. Paris: José Corti, 2004. . Adieux au poème. Paris: José Corti, 2005.

RENARD, Jean-Claude. Notes sur la poésie. Paris: Seuil, 1970.

RIMBAUD, Arthur. "Lettre à Paul Demeny ». In : RIMBAUD, Arthur. Poésies. Une saison en enfer. Illuminations. Paris: Gallimard, 1999.

TARDIEU, Jean. Margeries. Paris: Gallimard, 2009. 
VALÉRY, Paul. « Poésie et pensée abstraite ". In : VALÉRY, Paul. Variété III, IV et V. Paris: Gallimard, 2002. p. 567-691.

. Variété III, IV et V. Paris: Gallimard, 2002.

Recebido: 30/01/2018

Aceito: 25/07/2018

DOI - http://dx.doi.org/10.5902/2179219431001 